\title{
Seismic Linear Noise Attenuation with Use of Radial Transform
}

\author{
Żaneta Szymańska-Małysa ${ }^{1, *}$ \\ ${ }^{1}$ AGH University of Science and Technology, Faculty of Geology, Geophysics and Environmental \\ Protection, A. Mickiewicza Av. 30, 30-059 Krakow, Poland
}

\begin{abstract}
One of the goals of seismic data processing is to attenuate the recorded noise in order to enable correct interpretation of the image. Radial transform has been used as a very effective tool in the attenuation of various types of linear noise, both numerical and real (such as ground roll, direct waves, head waves, guided waves etc). The result of transformation from offset - time $(X-T)$ domain into apparent velocity - time $(R-T)$ domain is frequency separation between reflections and linear events. In this article synthetic and real seismic shot gathers were examined. One example was targeted at far offset area of dataset where reflections and noise had similar apparent velocities and frequency bands. Another example was a result of elastic modelling where linear artefacts were produced. Bandpass filtering and scaling operation executed in radial domain attenuated all discussed types of linear noise very effectively. After noise reduction all further processing steps reveal better results, especially velocity analysis, migration and stacking. In all presented cases signal-to-noise ratio was significantly increased and reflections covered previously by noise were revealed. Power spectra of filtered seismic records preserved real dynamics of reflections. Key words - seismic, noise attenuation, radial transform.
\end{abstract}

\section{Introduction}

Recording of seismic data always goes hand in hand with recording of unwanted noise. One of the goals of seismic processing is to attenuate the noise in the simplest and fastest way to enable correct interpretation of the image. The large group of unwanted energy constitutes linear noise, such as ground roll, air waves, direct waves, head waves, guided waves etc. After noise filtration all further processing steps become more reliable, especially deconvolution, velocity analysis, migration and stacking. Therefore proper signal-to-noise ratio improvement is so important.

In standard processing flow few transformations are used to treat such type of energy. Among the available tools the most popular are methods based on velocity, amplitude and / or frequency difference between reflections and noise. Author used radial transform $(R-T$ transform, apparent velocity - time transform) because presented method offered effective attenuation of all the linear noise present in data in one processing step, although it could also be used in iterative and / or adaptive mode.

\footnotetext{
* Corresponding author: zmalysa@agh.edu.pl
} 
Radial transform was first used in migration and imaging techniques [1-2]. Attenuation of multiples was another early implementation of use of $T-R$ domain [3-4]. Although coherent noise attenuation is general application of $R-T$ transform, recently described for instance by [5-7], it has become useful in other seismic fields, as for example seismic data interpolation [8]. Filtration in radial domain has formerly been studied by the author [9-10].

\section{Mathematical background}

Mathematical background of radial transform was discussed in detail for example in [11-13]. Wavefield amplitudes from gather of seismic traces $S$ with respect to radial origin $\left(x_{0}, t_{0}\right)$ are being extracted and interpolated into coordinates of apparent velocity $V$ and time $t^{\prime}$ (radial domain).

The $R-T$ transform is defined by:

$$
R[S(x, t)]=S^{\prime}\left(V, t^{\prime}\right)
$$

where $x$ is the source-receiver offset, $t$ is two-way travel time,

$$
V=\left(x-x_{0}\right) /\left(t-t_{0}\right) \text { and } t^{\prime}=t-t_{0} .
$$

The inverse radial transform is given by:

$$
R^{-1}\left[S^{\prime}\left(V, t^{\prime}\right)\right]=S(x, t)
$$

The result of radial transform is frequency separation between reflections and linear events. A sloping event which originates from the $R-T$ origin becomes vertical in the radial trace domain and is situated in narrow group of constant-velocity traces in the $R-T$ domain [13]. Furthermore, its temporal apparent frequency decreases significantly, while reflections, which do not pass through the $R-T$ origin, are almost unchanged in amplitude and phase. Bandpass filtering aimed at low frequency attenuation executed in radial domain attenuates such noise very effectively.

The important parameters of radial trace transform are: the interpolation method which influences the relative precision of remapping events in the $R-T$ domain [1], coordinates of the $R-T$ origin, apparent velocity range and type of transformation (radial fan transform or radial dip transform).

\section{Methodology}

Synthetic and real data were tested. The typical processing sequence consisted of: gather preconditioning (field statics - if necessary, normal moveut correction, top muting, gain - if necessary), forward radial transform, proper noise filtering and scaling, reverse radial transform.

Noise identification was carried out on the basis of apparent velocities and frequency bands in $X-T$ (offset-time) and $R-T$ (radial) domains. Power spectra of examined data have also been analyzed. To attenuate particular type of noise author measured its apparent velocity and examined its power spectrum in $X-T$ domain and $R-T$ domain.

Two modes of radial transform in used seismic software (Vista, Schlumberger) are available. Depending on type of noise and its strength so called 'fan' or 'dip' transform is used. The best option for the attenuation of ground roll is formation of fan of velocities, which cover the whole dataset, although this method can be used to attenuate any noise 
originating from the source. In this mode complete shot gather is transformed into $R-T$ domain and then processed. In radial domain one or set of bandpass filters followed by proper scaling can be used to attenuate stretched ground roll. In author's opinion this mode can be more "dangerous" because it converts the whole dataset, therefore may cause unwanted artifacts. To avoid distortion of reflections bandpass filter must be set very carefully. This results normally only in partial attenuation of noise.

Another available tool is dip radial filter which offers transformation of seismic amplitudes in limited range of apparent velocities. On the basis of apparent velocity of noise only very small portion of dataset can be processed, avoiding larger distortion of the signal. Then more "aggresive" bandpass filter can be used. Sometimes it is difficult to attenuate all linear events in one step because of their different apparent velocities and origins. By careful velocity and frequency range identification great precision in noise separation can be achieved.

\section{Results}

\subsection{Synthetic dataset}

First examined example is a set of seismic shot gathers which were produced by elastic modelling. On the top of Figure 1 first and last shot gathers in the dataset were shown.

\section{INPUT DATA}

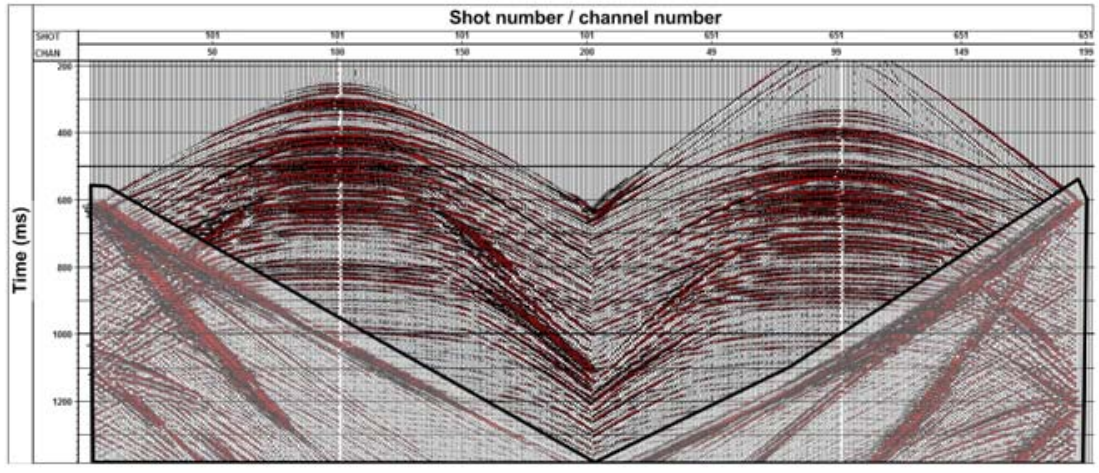

\section{OUTPUT DATA}

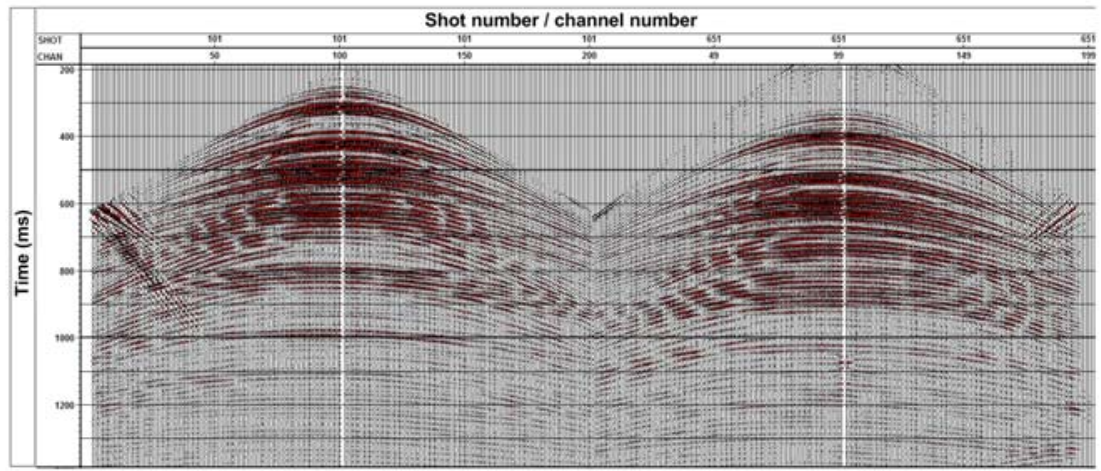

Fig. 1. Two synthetic shot records before (top picture) and after filtration in radial domain (bottom picture). Area of strong noise is marked on the top picture 
The linear artifacts produced by modelling (shaded area on the top of Fig. 1) are strong compared to seismic reflections and cover large area of the dataset. This type of noise is piecewise linear with relatively broad power spectrum shifted towards low frequencies. On the bottom of Figure 1 filtrated seismic record is shown.

In presented case the optimal solution was the use of two iterations of dip transform with proper filtration and scaling in the $R-T$ domain. To limit the negative influence of the transormation narrow range of velocities was used (1600-1800 m/s, positive and negative). The shot gather 101 from Figure 1 was transformed to the radial domain where high-amplitude noise was spread all over the data and had variable curvatures (left on Fig. 2), while reflections remained hyperbolic. Two iterations were conducted: first for positive velocities, second for negative velocities. Bandpass filtering set to attenuate low frequencies was performed to limit the noise and the result in radial domain is presented on the right on Figure 2. It is obvious that bandpass filter attenuated noise very effectively and left signal almost unchanged, therefore causing relative increase of signal amplitudes.

\section{FIRST ITERATION}

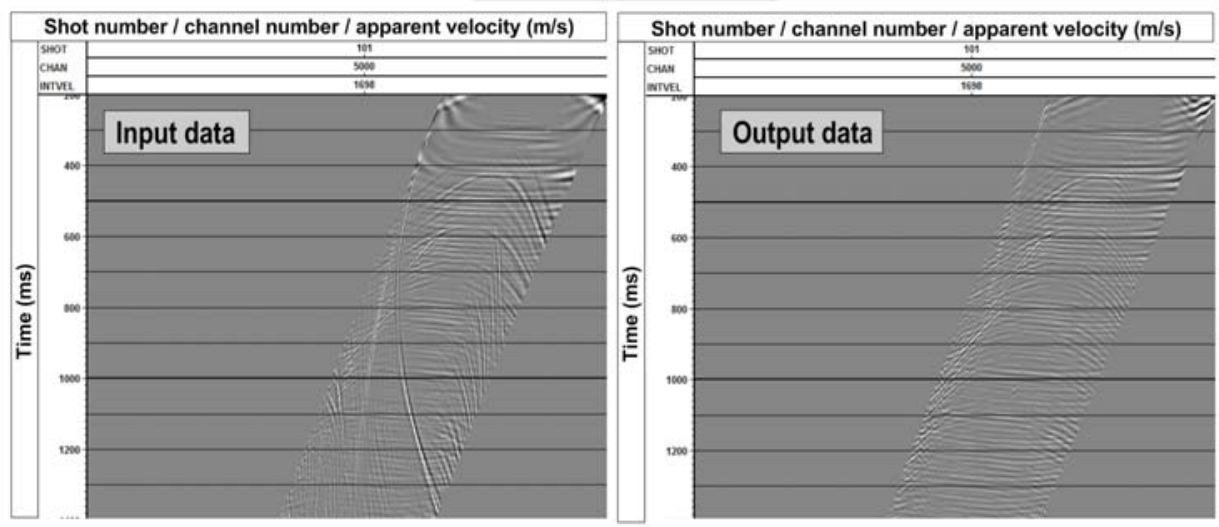

SECOND ITERATION

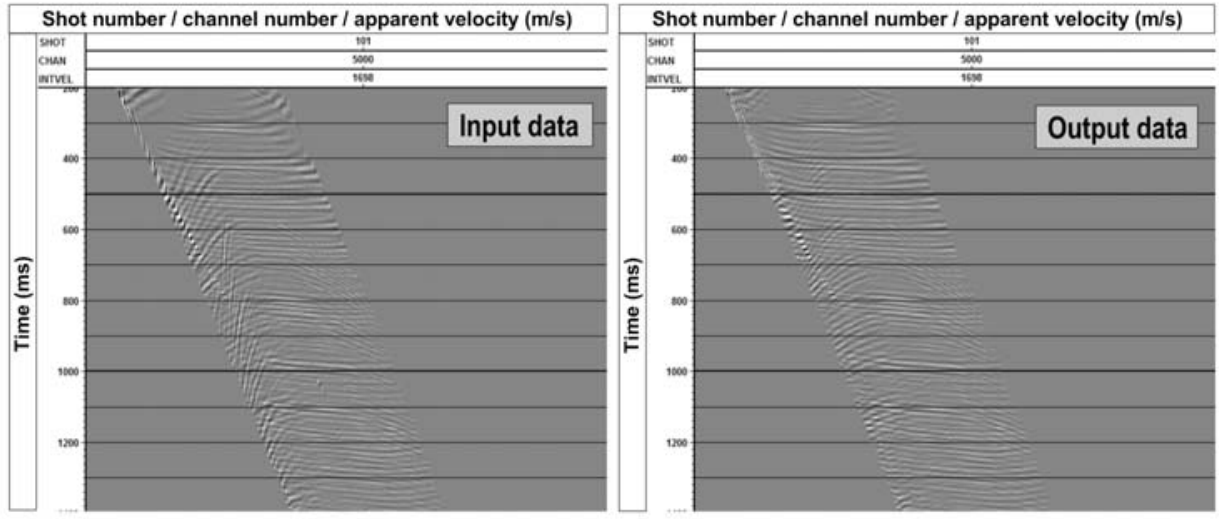

Fig. 2. Synthetic record in radial domain before and after first iteration (top picture) and after second iteration (bottom picture). Input record is shown on the left. The same record after first and second pass of bandpass filtration is shown on the right

When comparing the power spectra of input and filtered dataset (Fig. 3) it is clearly seen that proposed method left the signal almost unchanged except the area of low frequencies where noise dominated $(10-30 \mathrm{~Hz})$. In this zone power spectrum was slightly attenuated. 


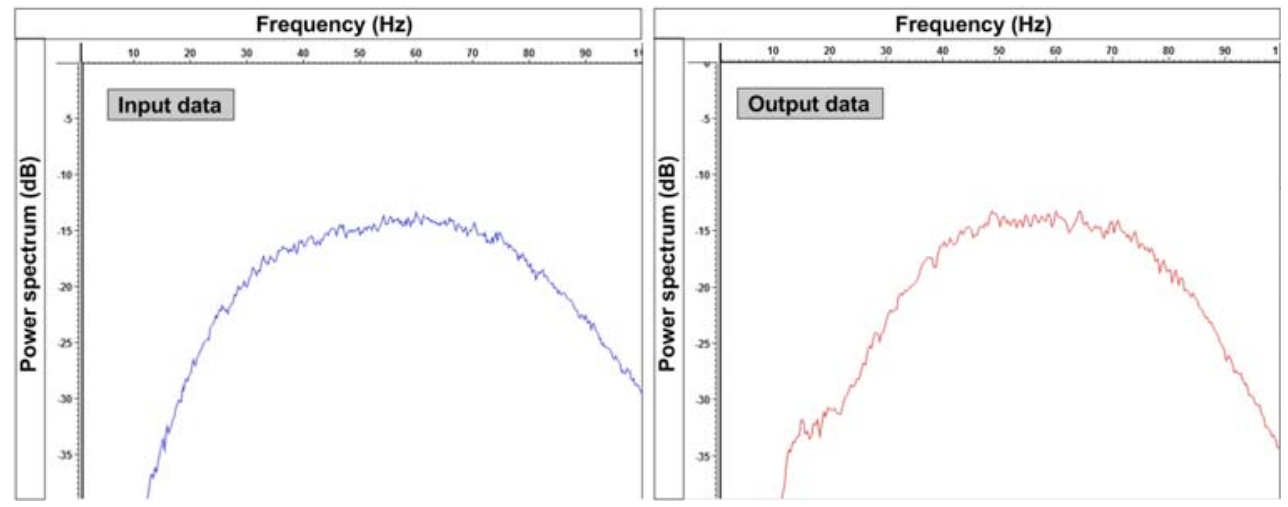

Fig. 3. Power spectrum of synthetic shot record before (left picture) and after sets of radial filters (right picture)

\subsection{Real dataset}

Another tests were conducted on land shot gathers acquired in Poland where very poor signal-to-noise ratio was observed. The presented real dataset was targeted on far offsets where reflections and noise had similar apparent velocities and frequency bands. At the top of the Figure 4 the far offset part of original shot record with its power spectrum is presented. The shot gather consists of reflections (very weak and almost invisible on raw data) and various types of linear noise: ground roll, direct wave, first breaks (refraction) and guided waves. Unwanted energy is here extremely strong and various filtration methods were examined to treat such kind of energy. The final results of use of radial transform were compared to results received from more common methods such as Radon (tau-p) transform and $f-k$ filter (second and third from the top on the Figure 4, respectively). The shot gathers and their power spectra were compared.

Linear Radon transform (tau-p transform) was performed to achieve noise model which was then adaptively subtracted from the shot gathers. Because transformation introduced some high-frequency noise additional bandpass filter was applied. It can be concluded from Figure 4 that such filtration caused attenuation of many of visible types of noise. Despite the fact that weak reflections became more evident (in particular reflection at $1900 \mathrm{~ms}$ ), it was not as successful as proposed method based on radial transform.

Data transformed to the $f-k$ domain revealed the aliasing of noise caused both by too sparse receiver interval and very low apparent velocity of ground roll. Designed filter has been found ineffective in noise attenuation except ground roll case. The aliasing was the reason why data became smeared and distorted, while noise was still strong and covered reflections.

Radial transform was performed in three steps by means of both fan and dip transform. First ground roll was attenuated. Afterwards other types of noise were handled. On the bottom of the Figure 4 record after radial transform with its power spectrum is shown. From the wavefield large amount of noise vanished, consequently improving the visibility of reflections at times 1100-1200 ms, $1700 \mathrm{~ms}$ and 1900-2000 ms. When comparing power spectra on Figure 4 it can be seen that every filtration method attenuated large amount of low frequency energy (10-30 Hz corresponding to ground roll). Other types of noise which were spread all over the signal spectrum were effectively attenuated only by means of radial method. 


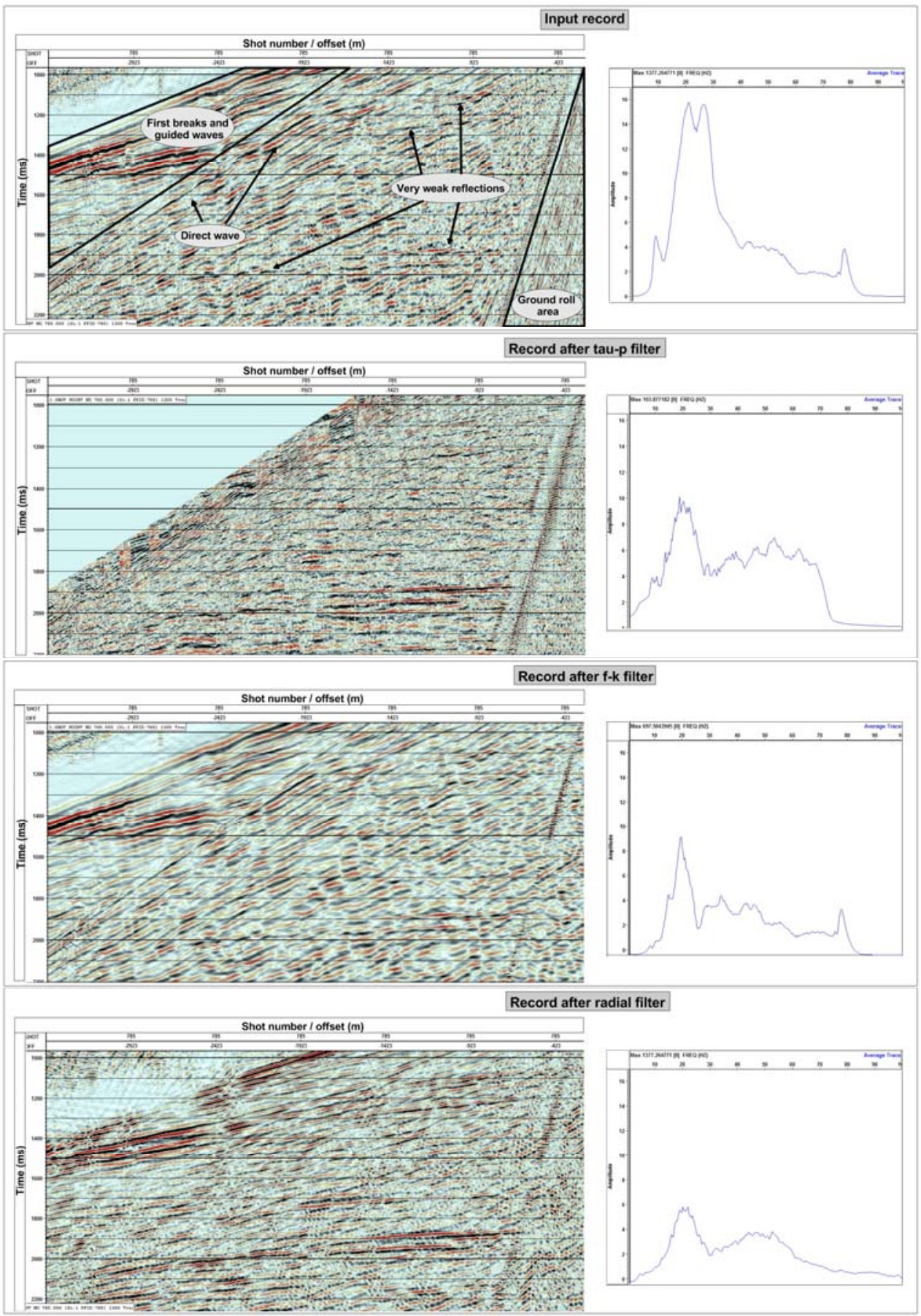

Fig. 4. Comparison of different filtration methods - shot gathers (on left) and the corresponding power spectra (on right)

One of the best evidence of importance of noise attenuation is creation of semblance functions which are used to map stacking velocity field (Fig. 5). On the left on Figure 5 raw semblance function was created. Velocity picking can not be executed with high reliability 
because the image is smeared. Set of filters executed in radial domain improved semblance image so much that velocity picking has become clear and easy (energy is focused to red dots on the right picture on Fig. 5).

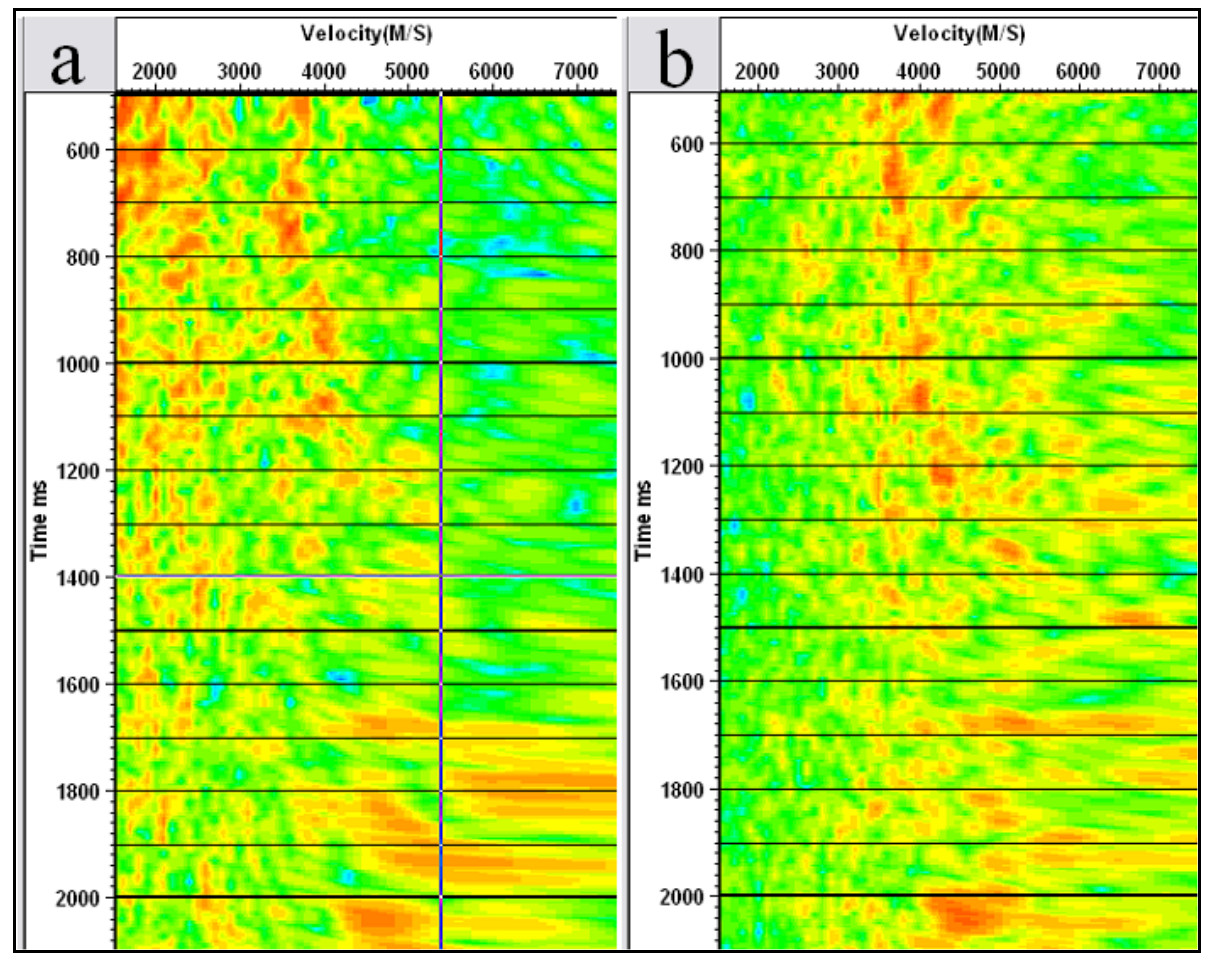

Fig. 5. Semblance function created on raw data (on the left) and the same function created on filtrated shot records (right)

\section{Conclusions}

Bandpass filtering in the radial domain enables effective and easy way of attenuation of various types of linear noise observed on seismic data. In all presented cases signal-to-noise ratio was significantly increased and reflections covered previously by noise were revealed. Power spectra of presented data are very little distorted and reveal real dynamics of reflections.

Radial transform performed in VISTA software (Schlumberger) presents many advantages with respect to other transforms, which mainly are: simplicity of the algorithm, ability to track every type of linear event, little distortion of reflections. Another advantages are: efficiency in transformation of irregularly spatially sampled data and ability to cascade the process to better treat particular type of noise. In author's opinion the most important advantage is the ability to attenuate wide range of generated linear noise in one step, even without additional data preconditioning or using adaptive noise attenuation algorithms.

Presented method reveals also some disadvantages. Among them the most important in author's opinion are: long computational time (the computational time increases with the amount of radial traces used), weakness in case of dispersive or non-linear events attenuation (they become smeared in radial domain and filtration is not so efficient). Because of amplitude balancing in radial domain, which generated better images, discussed results can not be used in AVO analysis. Without this special amplitude treatment results of filtration are little worse but also maintain high value. 
On the basis of conducted research author concludes that among presented methods radial transform eliminated wide range of noise in relatively fast and easy way while preserving original frequencies of signal. The presented examples prove that proper noise filtration and scaling in radial domain is competitive and effective way of noise elimination, guaranteeing better image interpretation.

\section{References}

1. J.F. Claerbout, Stanford Exploration Project Report, 35, 43-53 (1983)

2. R. Ottolini, Stanford Exploration Project Report, 20, 97-115 (1979)

3. M.T. Taner, Geophys. Prosp. 28(1), 30-48 (1980)

4. M.G. Lamont, B.M. Hartley, N.F. Uren, The Leading Edge 18(1), 110-114 (1999)

5. L.J.A. Almeida, R.R. Manenti, M.J. Porsani, Coherent Noise Attenuation using the Wavelet Transform on Radial Basis, SEG Technical Program Expanded Abstracts 2015, 4720-4724 (2015)

6. O.F. Adizua, J.O. Obeniro, C.N. Ehirim, Int. J. Res. Innov. Earth Sci, 3, 11-14 (2015)

7. W.L. Wang, W.Y. Yang, X.J. Wei, X. He, Appl. Geophys, 14(1), 96-104 (2017)

8. M. Zhang, Y. Liu, Seismic data Interpolation based on radial basis function, International Geophysical Conference, Quingdao, China, 17-20 April 2017, 435-439 (2017)

9. Ż. Szymańska-Małysa, Radial transform potential of coherent linear noise attenuation, 75th EAGE conference \& exhibition incorporating SPE EUROPEC 2013, London, UK, 10-13 June 2013 (2013)

10. Ż. Szymańska-Małysa, Seismic image improvement developed on the basis of radial transform, IX Krakow conference of young scientists 2014, Krakow, Poland, 2-4 October 2014, book of abstracts (2014)

11. D.C. Henley, The radial trace transform: An effective domain for coherent noise attenuation and wavefield separation, SEG Technical Program Expanded Abstracts 1999, 1204-1207 (1999)

12. D.C. Henley, Wavefield separation and other useful applications in the radial trace domain, SEG Technical Program Expanded Abstracts 2000, 2111-2114 (2000)

13. D.C. Henley, CSEG Recorder, 26(2), 21-29 (2001) 